Supporting Information for

\title{
Lymphatic Vessel on a Chip with Capability for Exposure to Cyclic Fluidic Flow
}

Parinaz Fathi, Glenn Holland, Dipanjan Pan, and Mandy B. Esch*

*mandy.esch@nist.gov 


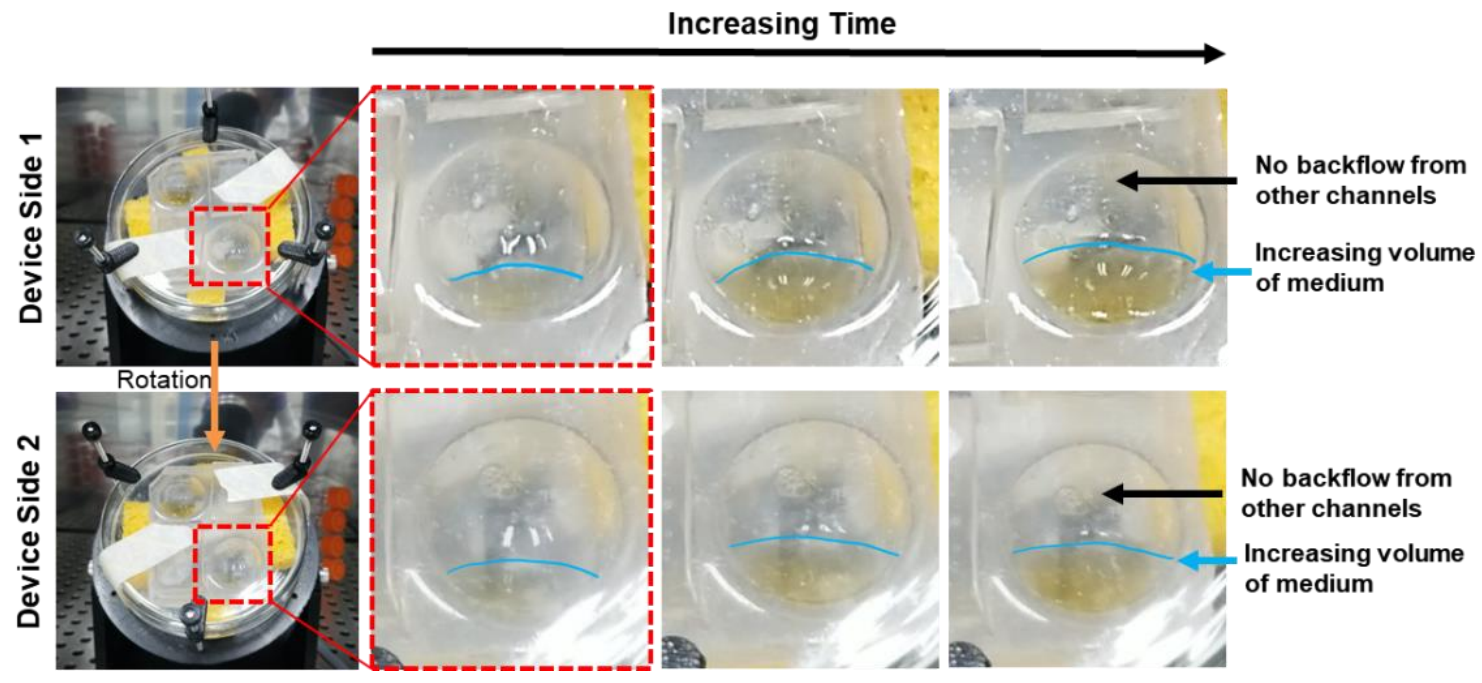

Figure S1. Screenshots from Supplementary Video 1, demonstrating unidirectional flow in the microfluidic device. An increase in the amount of media in the bottom hemispherical well is observed as a result of flow. Capillary forces prevent backflow from channels on the opposite side of the device. Rotation of the device results in cessation of flow in one set of channels and allows for flow in the other set of channels. 

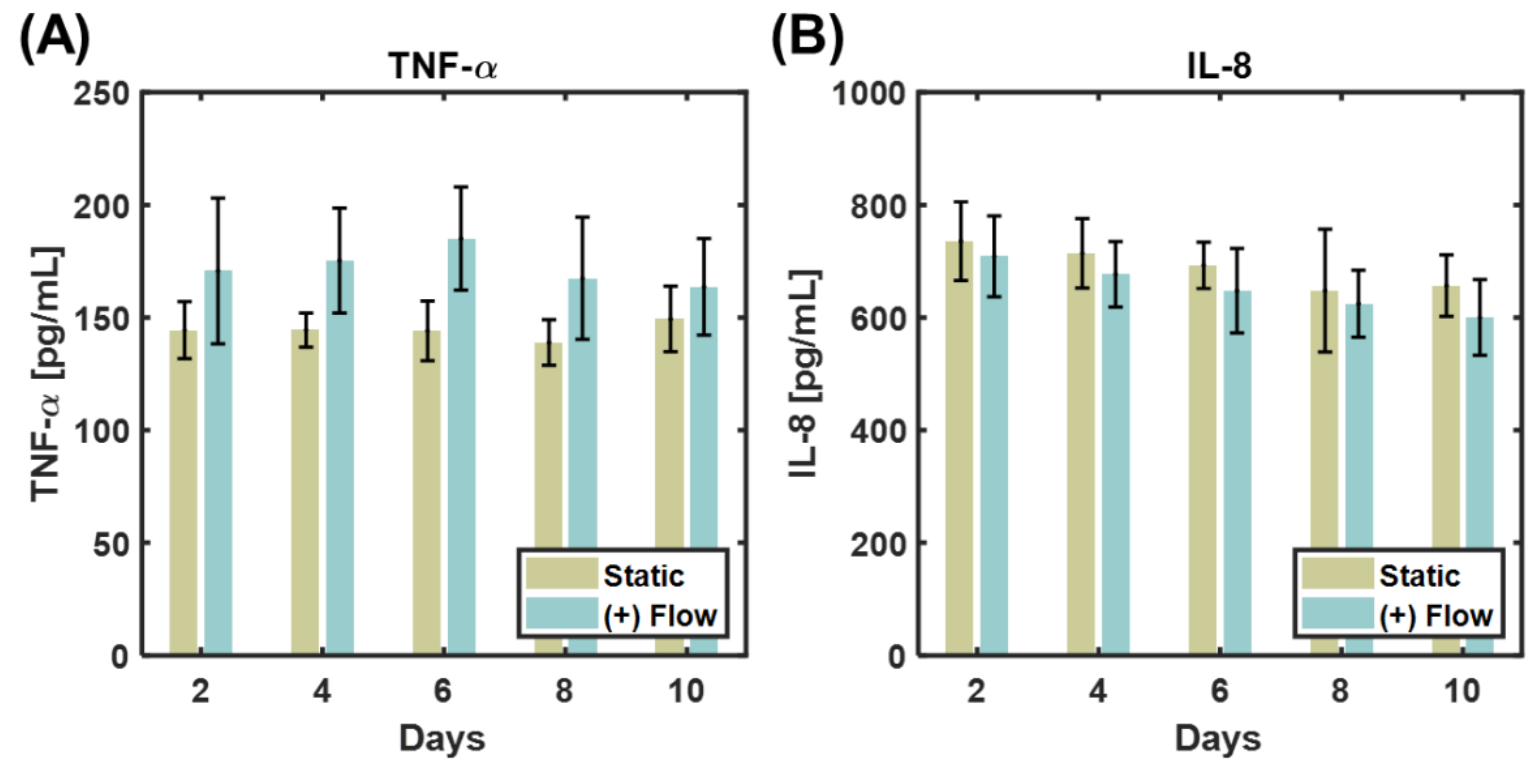

Figure S2. Non-normalized cytokine expression. (A)TNF- $\alpha$ expression, indicating no significant differences in devices with flow and without flow. (B) IL-8 expression, indicating no significant differences in devices with flow and without flow. Values are presented as averages, with error bars representing standard deviation across 8 devices (flow condition) or 7 devices (static condition). 